\title{
USO DE CATALISADOR MAGNÉTICO DO TIPO FERRITA NÍQUEL- ZINCO PARA A OBTENÇÃO DE BIODIESEL
}

\section{USE OF MAGNETIC CATALYST OF THE NICKEL-ZINC FERRITE TYPE TO OBTAIN BIODIESEL}

\section{R. L. N. GONÇALVES ${ }^{1 *}$, A. C. F. M. $\operatorname{COSTA}^{2}$ e K. R. O. PEREIRA ${ }^{2}$}

${ }^{1}$ Universidade Federal de Campina Grande, Unidade Acadêmica de Engenharia de Petróleo, Campina Grande, Paraíba, Brasil

${ }^{2}$ Universidade Federal de Campina Grande, Unidade Acadêmica de Engenharia de Materiais, Campina Grande, Paraíba, Brasil

${ }^{*}$ Corresponding author. Federal University of Campina Grande, Unidade Acadêmica de Engenharia de Petróleo, Campina Grande, Paraíba, Brasil, Phone: +55 83988969873

e-mail address: ruthlunang@gmail.com (R. L.N. Gonçalves).

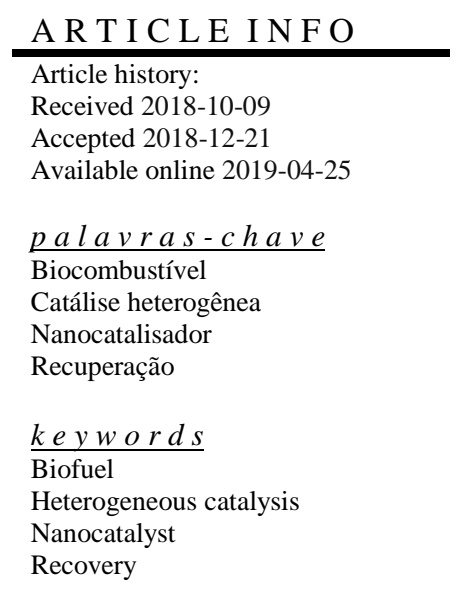

\begin{abstract}
A B S T R A C T
The reactions for biodiesel synthesis can be homogeneous or heterogeneous, depending on the catalyst used, being the heterogeneous reaction the best alternative because it allows the recovery of the catalyst at the end of the reaction. The aim of this paper was to analyze the ferrite type magnetic catalyst $\left(\mathrm{Ni}_{0.5} \mathrm{Zn}_{0.5} \mathrm{Fe}_{2} \mathrm{O}_{4}\right)$ efficiency, in a transesterification reaction, before and after its recovery. The biodiesel conversion rate was determined by gas chromatography. The results showed that, in the third reuse, the conversion rate dropped from $80.25 \%$ (virgin catalyst sample) to $68.31 \%$. That indicates that the recovery and subsequent reuse is viable, because the catalytic characteristics can still be maintained.
\end{abstract}

R E S UM O

As reações para a síntese de biodiesel podem ser homogêneas ou heterogêneas, a depender do catalisador utilizado, sendo a heterogênea a melhor alternativa por permitir que o catalisador seja recuperado após a reação. Este trabalho teve como objetivo analisar a eficiência do catalisador magnético do tipo ferrita $\left(\mathrm{Ni}_{0,5} \mathrm{Zn}_{0,5} \mathrm{Fe}_{2} \mathrm{O}_{4}\right)$, em reação de transesterificação, antes e após a sua recuperação. A taxa de conversão em biodiesel foi determinada por cromatografia gasosa. Os resultados mostraram que, no terceiro reuso, a taxa de conversão caiu de 80,25\% (amostra de catalisador virgem) para 68,31\%. Isto indica que a recuperação e posterior reuso é viável, pois as características catalíticas ainda podem ser mantidas. 


\section{INTRODUÇÃO}

O Brasil é líder global na utilização de biocombustíveis, particularmente etanol. A matriz energética brasileira avançada mostra que $43,2 \%$ da sua energia primária é proveniente de fontes renováveis. A oferta total de bioenergia em 2017 foi correspondente a $30 \%$ da matriz energética brasileira. Os produtos da cana (bagaço e etanol) responderam por $58,1 \%$ da bioenergia e por $17,4 \%$ da matriz. Outras bioenergias (lixívia, biogás, resíduos de madeira, resíduos da agroindústria e biodiesel) responderam por $15,2 \%$ da bioenergia e por $4,6 \%$ da matriz. (BRASIL, 2018). Uma característica importante dos biocombustíveis é ser proveniente da biomassa, a qual pode ser definida como toda matéria viva ou morta oriunda de plantas, animais ou seus derivados. Esta característica permite o uso integral de matérias-primas brutas, beneficiadas ou residuais para a produção de biocombustíveis, o que permite a redução da poluição do solo, do ar e de mananciais aquíferos, gerar um nicho de renda para comunidades carentes, agregar valor à matérias-primas de baixo valor, entre outros (DEPHE e FUKUOKA, 2008).

Dentre esses biocombustíveis, o biodiesel é o que vem recebendo uma séria atenção global, pois é um potencial combustível substituto ao diesel mineral, parcialmente ou completamente (AGARWAL et al., 2017).

De acordo com a Resolução ${ }^{\circ} 23$ do Conselho Nacional de Política Energética (CNPE), em março de 2018 a composição de biodiesel ao óleo diesel vendido ao consumidor subiu para 10\% (B10). O Brasil foi o segundo maior mercado de biodiesel em 2017, seguindo os Estados Unidos, com 7,4 bilhões de litros. O Centro-Oeste e o Sul foram as regiões com maior produção, responsáveis por $82 \%$ do total (GOVERNO DO BRASIL, 2018).

A obtenção do biodiesel, seja pela reação de transesterificação ou esterificação, é o processo mais usado e se dá pela inserção de três componentes, a saber: álcool (metanol e/ou etanol), óleo vegetal ou gordura animal e catalisador (ARAÚJO et al., 2016).

Processos heterogêneos devem apresentar vantagens técnicas, econômicas e ambientais em relação aos processos homogêneos convencionais, principalmente por haver a possibilidade da redução do número de etapas de purificação do biodiesel, facilidades na recuperação e purificação da glicerina, reutilização do catalisador e menor geração de efluentes, diminuindo assim o custo financeiro e ambiental da produção (CORDEIRO et al., 2011).

A catálise heterogênea possibilita o uso de álcoois de elevado peso molecular e em alguns casos, conseguem alcançar altos valores de conversão em sistemas, nos quais catalisadores homogêneos são inativos (HE e SCHOENUNG, 2002; SUAREZ et al., 2007). Também, os catalisadores podem ser reutilizados e consequentemente não ser descartado para o meio ambiente. Daí o interesse em substituir os catalisadores homogêneos pelos catalisadores heterogêneos, devido os procedimentos operacionais mais fáceis e a redução

Em seguida acomodado em recipientes para caracterização.

\subsection{Caracterização dos Produtos Reacionais}

Os produtos resultantes da reação foram analisados significativa da poluição ambiental (SCHUCHARDT, 2006).

Tal substituição vem se destacando mediante a utilização de materiais magnéticos nanoestruturados, que vem sendo apontado como uma nova possibilidade de uso aos materiais já existentes, bem como na descoberta de novos materiais. Em especial, a utilização de nanopartículas magnéticas em catálise heterogênea abre um leque de oportunidades científicoeconômicas em função de alguns problemas em aberto, que cercam tal aplicação, como por exemplo: a determinação de métodos mais eficazes e de custo mais baixo para sua obtenção em larga escala, bem como dos possíveis ganhos de escala almejados em novos processos industriais que empreguem catalisadores nanoestruturados (BRESSIANE et al., 2009).

De acordo com o exposto, este trabalho buscou determinar a eficiência do catalisador recuperado (nanopartícula magnética (NPM) do tipo (Ni-Zn) $\mathrm{Fe}_{2} \mathrm{O}_{4}$ obtida por reação de combustão) para uso em reação para obtenção de biodiesel.

\section{METODOLOGIA}

Foi utilizado como catalisador a nanopartícula magnética do tipo $\mathrm{Ni}_{0,5} \mathrm{Zn}_{0,5} \mathrm{Fe}_{2} \mathrm{O}_{4}$, selecionada em estudos anteriores (DANTAS et al., 2015, PEREIRA et al. 2015 e PEREIRA et al., 2014), bem como o tipo de reação e condições reacionais para obtenção do biodiesel.

Para as reações de transesterificação pela rota metílica foram utilizados os seguintes materiais: óleo de soja comercial e álcool metílico P.A (concentração 99,9\%).

As etapas do trabalho experimental consistiram em:

- Realizar processo catalítico com catalisador virgem (ou principal);

- Verificar as melhores condições para recuperação do catalisador;

- Reutilizar o catalisador.

\subsection{Testes Catalíticos (síntese do biodiesel com catalisador virgem)}

Os testes catalíticos foram conduzidos em reator de aço inox e a reação foi executada nas seguintes condições:

- $\quad$ Temperatura $=160^{\circ} \mathrm{C}$;

- Razão molar álcool:óleo = 1:9;

- $\quad$ Quantidade de catalisador $=2 \%$;

- Tempo de reação $=1$ hora.

Inicialmente pesou-se cada material a ser utilizado na reação, como: óleo, álcool e catalisador, que foram condicionados em copo tipo Becker e disposto no reator. Após o tempo de reação, esperou-se que o reator atingisse temperatura ambiente. O biodiesel obtido foi transferido para um funil de separação e lavado sucessivamente com água destilada para separação do óleo.

Após lavagem, o óleo obtido foi acondicionado em cubetas e centrifugado a 9000 RPM durante 20 minutos. Este procedimento foi necessário para separação completa do óleo.

quanto a conversão (C) em éster metílico, por cromatografia a gás usando-se um cromatográfico VARIAN 450c com detector de ionização de chama (FID) - (Flame Ionization Detection) em uma coluna capilar curta DB1 da J\&W Scientific. A temperatura do detector foi de $250{ }^{\circ} \mathrm{C}$ e a do 
injetor de $240^{\circ} \mathrm{C}$. A temperatura do forno foi programada de 150 até $260^{\circ} \mathrm{C}$ a uma taxa de aquecimento de $10^{\circ} \mathrm{C} / \mathrm{min}$. O gás de arraste empregado foi $\mathrm{H}_{2}$ ultrapuro.

As amostras de óleo foram preparadas da seguinte forma: diluição de $50 \mathrm{mg}$ do óleo (obtido na reação) em $5 \mathrm{~mL}$ de n-hexano padrão UV-HPLC e posterior injeção de um microlitro desta solução.

\subsection{Recuperação do catalisador}

O catalisador separado da mistura foi lavado com
Hexano em Becker e o excesso de solvente foi retirado. $\mathrm{O}$ catalisador foi seco a temperatura ambiente por 10 minutos, sendo armazenado para posterior reuso.

\section{RESULTADOS E DISCUSSÕES}

Os resultados de conversão em ésteres obtidos para o catalisador virgem e recuperado são apresentados na Tabela 1.

Tabela 1 - Conversão em biodiesel das amostras utilizando catalisador virgem e recuperado.

\begin{tabular}{|c|c|c|c|c|}
\hline Catalisador & Principal & $1^{\circ}$ reuso & $2^{\circ}$ reuso & $3^{\circ}$ reuso \\
\hline Conversão/\% & 80,25 & 73,3 & 64,24 & 68,31 \\
\hline
\end{tabular}

A partir dos resultados apresentados na Tabela 1, é possível verificar que a amostra principal (catalisador virgem) apresentou conversão de $80,25 \%$, e as amostras de reuso apresentaram $73,3 \%, 64,24 \%$ e $68,31 \%$, no $1^{\circ}, 2^{\circ}$ e $3^{\circ}$ reuso, respectivamente. A partir dos dados da Tabela 1, foi plotado um gráfico de colunas, mostrado na Figura 1, representando a taxa de conversão em biodiesel e suas respectivas amostras.

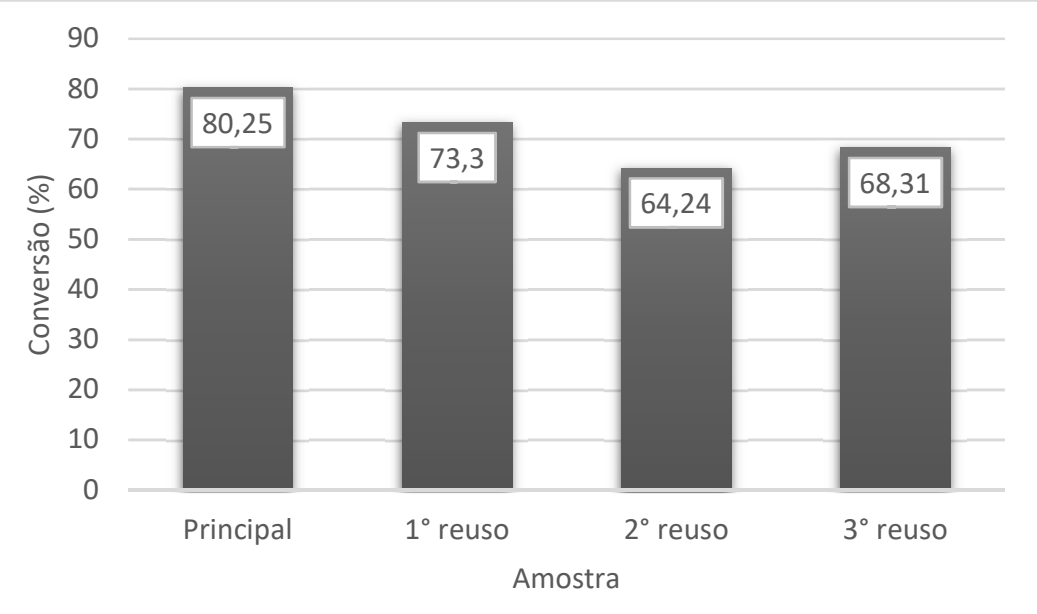

Figura 1 - Taxa de conversão de biodiesel utilizando catalisador magnético recuperado.

De acordo com os dados, pode-se notar a coerência dos resultados obtidos, pois à medida que o catalisador foi sendo reutilizado, ele foi perdendo a sua eficiência.

Diversos catalisadores já foram sintetizados e avaliados na obtenção de biodiesel por meio da reação de transesterificação ou esterificação de óleos vegetais ou gordura animal. Dentre eles pode-se citar:

Singh, Yadav e Sharma et al. (2017) sintetizaram aluminato bicálcico $\left(\mathrm{Ca}_{2} \mathrm{Al}_{2} \mathrm{O}_{5}\right)$ pelo método sólido usando carbonato de cálcio e alumina calcinada a $900{ }^{\circ} \mathrm{C}$. Este catalisador foi utilizado para a produção de biodiesel usando óleo de fritura vegetal e metanol através da transesterificação. Acetona foi usada e reportada como cosolvente pela primeira vez para a síntese de biodiesel. A maior taxa de conversão $(97,98 \%)$ foi obtida com uma porcentagem de $20 \%$ de acetona, razão-molar (óleo:metanol) de 1:6, porcentagem de $1,2 \%$ de catalisador. A temperatura e o tempo de reação foram de $55{ }^{\circ} \mathrm{C}$ e 25 minutos, respectivamente. $\mathrm{O}$ catalisador pôde ser reutilizado até oito vezes com uma taxa de conversão acima de $75 \%$.

Kaur, Malhotra e Ali (2018) sintetizaram tungstênio em formato de flor suportado em $\mathrm{TiO}_{2} / \mathrm{SiO}_{2}$ para ser usado como catalisador no processo de transesterificação de triglicerídeos. Sob condições ótimas de reação $(5 \%$ de $20 \mathrm{~W} / \mathrm{TiO}_{2} / \mathrm{SiO}_{2}-700$, razão molar metanol-óleo de 30:1 e temperatura de reação de $65^{\circ} \mathrm{C}$ ) a taxa de conversão obtida foi maior que $98 \%$ para um tempo de 4 horas. $\mathrm{O}$ catalisador foi reciclado e reutilizado em quatro ciclos consecutivos sem perdas significativas na eficiência e no quinto ciclo apresentou perda de $50 \%$ de eficiência.

\section{CONCLUSÃO}

A partir dos estudos e de práticas laboratoriais foi possível determinar uma rota adequada para a recuperação do catalisador, com a menor perda de material possível.

Foi determinado que o catalisador perde a sua eficiência à medida que é reutilizado, chegando a uma taxa de perda de $14,8 \%$ entre o catalisador virgem e o último reuso.

É possível observar que os resultados obtidos neste trabalho estão em concordância com estudos citados em literatura. 


\section{AGRADECIMENTOS}

O presente trabalho foi realizado com apoio do $\mathrm{CNPq}$, Conselho Nacional de Desenvolvimento Científico e Tecnológico - Brasil.

\section{R E F E R E N C I A S}

AGARWAL, A. K.; GUPTA, J. G.; DHAR, A. Potential and challenges for large-scale application of biodiesel in automotive sector. Progress in Energy and Combustion Science, vol. 61, pp. 113 - 149, 2017.

ARANDA, D. A. G.; GONÇALVES, J. A.; PERES, J. S.; RAMOS, A. L. D.; MELO JR, C. A. R.; The use of acids, niobium oxide, and zeolite catalysts for esterification reactions. Journal of Physical Organic Chemistry, Chichester, Inglaterra, GB, v. 22, p. 709-716, 2009.

ARAÚJO, N. O.; PEREIRA, K. R. de O.; BARROS, A. B. de S.; MOURA, T. F. B.; VILAR, E.; DANTAS, J.; COSTA, A. C. F. de M. Desempenho de catalisador magnético em esterificação metílica do óleo de soja. In: Congresso Brasileiro De Engenharia E Ciência Dos Materiais, 22., 2016, Natal. Anais. Natal: Congresso Brasileiro de Engenharia e Ciência dos Materiais, 2016. p. 1898 - 1907.

BRASIL. Ministério de Minas e Energia - MME. Resenha energética brasileira. Brasília, junho de 2018. Disponível em: <http://www.mme.gov.br/documents/10584/35804 98/02+-+Resenha+Energética+Brasileira+2018++ano+ref.+2017+\%28PDF\%29/6f00cc7c-be9a4257-ab5d8ab1935611aa;jsessionid=DE9158023A984B962 AECA56C0CB6595C.srv155> Acesso em: 02/07/2018.

BRESSIANI, J. C.; FONSECA, F. C.; SERRA, E. T.; FRAJNDLICH, E. U. C. Estudo Prospectivo de Materiais Avançados. Materiais Avançados para Energia no Brasil 2010-2020. Centro de Gestão e Estudos Estratégicos. Brasília - DF, 2009.

COLOMBO, K.; BARROS, A. A. C. Utilização de catalisadores heterogêneos na produção de Biodiesel. Evidência, v. 9, n. 1-2, p. 7-16, 2009.

CORDEIRO, C. S.; SILVA, F. R.; WYPYCH, F.; Ramos L. P. Catalisadores heterogêneos para a produção de monoésteres graxos (biodiesel). Química Nova, 34 - 3, 477-486, 2011.

DANTAS, J.; SILVA, F. N.; PEREIRA, K. R. O.; SILVA, A. S.; COSTA, A. C. F. de M. Evaluation of the Processing Conditions in the Transesterification for Biodiesel Production using the Nanomagnetic Catalyst Ni0.5Zn0.5Fe2O4. Materials Science Forum, v. 820, p. 113-118, 2015.

DHEPE, P. L.; FUKUOKA, A. Cellulose Conversion under Heterogeneous Catalysis. ChemSusChem. Vol. 1, pp. 969-975, 2008.

GOVERNO DO BRASIL. Produção de biodiesel atinge maior patamar dos últimos dez anos. Disponível em:

$<$ http://www.brasil.gov.br/editoria/infraestrutura/2 018/05/producao-de-biodiesel-atinge-maiorpatamar-nos-ultimos-dez-anos> Acesso em: 02/07/2018.

HE, J.; SCHOENUNG, J. M. Nanostructured coatings. Materials Science and Engineering A. v, 336, p. 274-319, 2002.

KAUR, M.; MALHOTRA, R.; ALI, A. Tungsten supported $\mathrm{Ti} / \mathrm{SiO} 2$ nanoflowers as reusable heterogeneous catalyst for biodiesel production. Renewable Energy, [s.1.], v. 116, p.109-119, fev. 2018. Elsevier BV

PEREIRA, K. R. O.; DANTAS, J.; DUTRA, R. C. L.; DINIZ, M. F.; Silva, A. S.; COSTA, A. C. F. de M. Synthesis of the Magnetic Nanoferrites of Spinel Type and Use in Esterification Reaction for Biodiesel Obtaining. Materials Science Forum , v. 820, p. 125-130, 2015.

PEREIRA, K. R. O.; DANTAS, J.; Silva, A. S.; COSTA, A. C. F. de M. Síntese de Nanopartículas Magnéticas

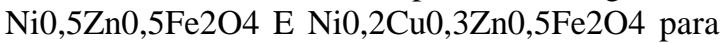
Obtenção de Biodiesel. In: $58^{\circ}$ Congresso Brasileiro de Cerâmica, 2014, Bento Gonçalves - RS. Anais do $58^{\circ}$ Congresso Brasileiro de Cerâmica, 2014.

SCHUCHARDT, U. L. F. Matérias-primas alternativas para a produção de biodiesel por catálise ácida. In: II Simpósio do Agronegócio de Plantas Oleaginosas, 2006.

SINGH, V.; YADAV, M.; SHARMA, Y. C.. Effect of cosolvent on biodiesel production using calcium aluminium oxide as a reusable catalyst and waste vegetable oil. Fuel, [s.1.], v. 203, p.360-369, set. 2017. Elsevier BV.

SUAREZ, P. A. Z.; MENEGHETTI, S. M. P.; MENEGHETTI, M. R.; WOLF, C. R. Transformação de triglicerídeos em combustíveis, materiais poliméricos e insumos químicos: Algumas aplicações da catálise na oleoquímica. Química Nova. v. 30, n. 3, p. 667-676, 2007.

VINES, T.; RIESEBERG, L.; SMITH, H. No crisis in supply of peer reviewers. Nature. vol. 468, 2010. 